\title{
硅烷偶联剂对制备硅酸锆薄膜及其抗氧化性能的影响
}

\author{
陈 婷 ${ }^{1,2}$, 查剑锐 ${ }^{1}$, 张篵君 ${ }^{1}$, 江伟辉 ${ }^{1,2}$, \\ 江 莞 $^{2}$, 刘健敏 ${ }^{2}$, 吴 倩 ${ }^{2}$
}

(1. 景德镇陶瓷大学 材料科学与工程学院, 景德镇 333004; 2. 景德镇陶瓷大学 国家日用及建筑陶瓷工程中心, 景德 镇 333001)

摘 要: 采用乙酸锆和正硅酸乙酯为原料, 硅烷偶联剂 3-氨丙基三乙氧基硅(APTES)为表面改性剂, 通过溶胶-凝胶 法制备了致密硅酸锆薄膜。系统研究了 APTES 的添加对硅酸锆薄膜的晶体结构、显微形貌及其抗氧化性能的影响。 实验结果表明, 添加硅酸锆摩尔浓度 $10 \mathrm{~mol} \%$ 的 APTES 可以制备出纯相的致密硅酸锆薄膜, 超过这一浓度则会出 现少量的 $\mathrm{SiO}_{2}$ 杂质, 同时造成硅酸锆溶胶的黏度迅速升高。AFM 测试结果表明添加 APTES 制备的薄膜更致密平 滑。 $1300^{\circ} \mathrm{C}$ 抗氧化测试 $102 \mathrm{~h}$ 后, 未镀膜的碳化硅基底增重 $0.16 \%$, 相比之下, 添加 APTES 前后的样品的增重分别为 $0.08 \%$ 和 $0.03 \%$, 说明添加 APTES 有利于提高 $\mathrm{ZrSiO}_{4}$ 薄膜的致密度, 进而提高其抗氧化性能。

关 键 词: 薄膜; 硅酸锆; 溶胶一凝胶法; 抗氧化

中图分类号: TB383 文献标识码: A

\section{Silane Coupling Agent on Synthesis and Antioxidation Property of Zircon Film}

\author{
CHEN Ting ${ }^{1,2}$, ZHA Jian-Rui ${ }^{1}$, ZHANG Xiao-Jun ${ }^{1}$, JIANG Wei-Hui ${ }^{1,2}$, \\ JIANG Wan ${ }^{2}$, LIU Jian-Min ${ }^{2}$, WU Qian ${ }^{1}$
}

(1. School of Material Science and Engineering, Jingdezhen Ceramic Institute, Jingdezhen 333004, China; 2. National Engineering Research Center for Domestic \& Building Ceramics, Jingdezhen 333001, China)

\begin{abstract}
The dense zircon film was prepared via Sol-Gel method using zirconium acetate and tetraethoxysilane as raw materials, and silane coupling agent 3-aminopropyltriethoxysilane (APTES) as surface modifying agent, respectively. Influences of APTES on the phase composition, morphology and antioxidation property of the $\mathrm{ZrSiO}_{4}$ film were investigated. The experiment results indicated that the pure and dense zircon film was achieved with addition of $10 \mathrm{~mol} \%$ APTES into $\mathrm{ZrSiO}_{4}$ sol. Once the molar ratio of APTES was beyond $10 \mathrm{~mol} \%$, the impurity $\mathrm{SiO}_{2}$ was observed, and the viscosity of the $\mathrm{ZrSiO}_{4}$ sol increased significantly at the same time. AFM results showed that the smooth and dense film was obtained with APTES addition. After $102 \mathrm{~h}$ oxidation cyclic test at $1300^{\circ} \mathrm{C}$, uncoated $\mathrm{SiC}$ substrate gained weight of $0.16 \%$, while samples coated with $\mathrm{ZrSiO}_{4}$ thin films only gained weight of $0.08 \%$ and $0.03 \%$ before and after addition of APTES. In summary, APTES addition is beneficial for the density of $\mathrm{ZrSiO}_{4}$ thin film, resulting in excellent anti-oxidation property.
\end{abstract}

Key words: thin film; zircon; Sol-Gel method; anti-oxidation

收稿日期: 2017-01-16; 收到修改稿日期：2017-03-09

基金项目：国家自然科学基金(51402135，51362014); 江西省科技计划项目(20151BBE50015); 江西省教育厅基金(GJJ150919, GJJ150935)

National Natural Science Foundation of China (51402135, 51362014); Projects of Jiangxi Provincial Department of Science and Technology (20151BBE50015); Science Foundation of Jiangxi Provincial Department of Education (GJJ150919, GJJ150935)

作者简介：陈 婷(1984-), 女，讲师. E-mail: chenting@jci.edu.cn

通讯作者: 江伟辉, 教授. E-mail: jiangweihui@jci.edu.cn; 江 莞, 教授. E-mail: wanjiang@dhu.edu.cn 
碳化硅材料具有优异的高温力学性能、高温强 度、热导率、抗蠕变性能和抗热震性能等特点, 在 冶金、化工和航空航天工业中具有广泛的应用前景 ${ }^{[1-2]}$ 。 作为一种典型非氧化物材料, 碳化硅的高温抗氧化 性能存在明显缺陷，水蒸气、碱、硫酸盐等物质都 会造成该材料的氧化，缩短其使用寿命 ${ }^{[3]}$ 。如何提高 碳化硅材料的抗氧化性成为目前亟待解决的重要问 题。除了改善其自身抗氧化性能外, 在碳化硅材料 表面涂覆一层抗氧化涂层或薄膜, 隔绝碳化硅与外 界氧气接触和反应是缓解氧化的有效方法之一 ${ }^{[3-4]}$ 。

硅酸锆薄膜具有较高的熔点、低氧渗透率、高 稳定性、与 $\mathrm{SiC}$ 良好的热膨胀匹配性 $\left(\mathrm{ZrSiO}_{4}\right.$ 的热膨胀 系数为 $4.1 \times 10^{-60} \mathrm{C}^{-1}, \mathrm{SiC}$ 的热膨胀系数为 $4.7 \times 10^{-6{ }^{\circ}} \mathrm{C}^{-1}$ ), 引起了科研工作者的广泛关注 ${ }^{[5-7]}$ 。目前, 硅酸锆薄 膜的制备方法主要有气相沉积法、水热电泳沉积法、 大气等离子喷涂法和溶胶-凝胶法等 ${ }^{[8-11]}$ 。Morais 等 ${ }^{[8]}$ 通过气相沉积法制备了大面积的硅酸锆薄膜, 但 制备的硅酸锆薄膜为非化学计量比化合物, 晶体稳 定性相对较低; Liu 等 ${ }^{[9-10]}$ 使用纳米粉体为原料, 通 过水热电泳沉积法在 $\mathrm{SiC}$ 基体表面制备了硅酸锆涂 层, 该涂层经 $1500^{\circ} \mathrm{C}$ 抗氧化测试 $332 \mathrm{~h}$ 后基体质量 损失仅为 $16.13 \mathrm{mg} / \mathrm{cm}^{2}$ 。溶胶一凝胶法是制备薄膜材 料的常用方法之一 ${ }^{[11-13]}$, 其过程主要是由无机盐或 金属醇盐经水解、缩聚形成凝胶网络, 随后将基片 提拉镀膜、煅烧后获得均匀致密的薄膜材料, 该法 具有样品均匀度高、易掺杂改性、反应过程易于控 制、设备要求低、溶剂易脱出等优点 ${ }^{[14]}$ 。此外, 决 定薄膜抗氧化性的因素除了材料本身性质以外, 还 与薄膜的缺陷(针孔、空洞和裂纹)以及膜/基界面特 征密切相关 ${ }^{[15]}$ 。因此可以通过改善薄膜和基体的附 着强度消除膜中的缺陷，从而有效提高其抗氧化性 能。本工作采用非水系溶剂乙酸为溶剂, 乙酸锆和 正硅酸乙酯为原料, 通过溶胶一凝胶法制备了硅酸 锆薄膜。系统研究了添加硅烷偶联剂(APTES)对提 高薄膜的抗氧化能力的影响。

\section{1 实验方法}

\section{1 硅酸锆薄膜的制备}

采用溶胶-凝胶法制备硅酸锆薄膜的过程如下: 量取 $0.02 \mathrm{~mol}$ 的正硅酸乙酯(TEOS)置于雉形瓶内, 随后加入矿化剂氟化锂 $(\mathrm{LiF})$ 与 TEOS 混合均匀得到 混合液, 其中 $\mathrm{Li} / \mathrm{Si}$ 摩尔比为 $3: 10$ 。同时, 按照 $\mathrm{Si}$ : $\mathrm{Zr}=1.2: 1$ 的摩尔比量取一定量的乙酸锆溶于乙酸 中, 调节溶胶前驱体浓度为 $0.4 \mathrm{~mol} / \mathrm{L}$, 随后加入与 乙酸锆的摩尔比为 $1: 1$ 的螯合剂乙酰丙酮。将以上
两种溶液混合至均匀, 滴加 $\mathrm{HNO}_{3}$ 调节 $\mathrm{pH}$ 至 1 , 随 后分别加入摩尔比为 $5 \% 、 10 \% 、 15 \% 、 20 \%$ 的硅烷 偶联剂(APTES), 最后置于室温自然陈化 $24 \mathrm{~h}$ 获得 硅酸锆溶胶。

将碳化硅基片交替使用硝酸和去离子水超声洗 净, 置于真空干燥箱中烘干后浸入硅酸锆溶胶中, 随后以 $1 \mathrm{~mm} / \mathrm{s}$ 的速度将基片平稳地从溶胶中提拉 出来, $60^{\circ} \mathrm{C}$ 真空干燥 $2 \mathrm{~h}$ 后获得干凝胶膜。重复 4 次 镀膜后热处理得到无开裂的硅酸锆薄膜: 首先从室 温以 $1^{\circ} \mathrm{C} / \mathrm{min}$ 速率升温至 $500^{\circ} \mathrm{C}$ 保温 $0.5 \mathrm{~h}$ 。随后以 $2^{\circ} \mathrm{C} / \mathrm{min}$ 速率升温至 $1000^{\circ} \mathrm{C}$ 保温 $1 \mathrm{~h}$, 自然冷却至室 温后获得硅酸锆薄膜。添加 APTES 辅助溶胶-凝胶 法制备 $\mathrm{ZrSiO}_{4}$ 的机理图如图 1 所示。APTES 中的 $\mathrm{NH}^{3+}$ 易与基片表面的 $\mathrm{OH}^{-}$产生正负电吸引，另一端 的 $\mathrm{Si}-\mathrm{O}$ 可以和 $\mathrm{ZrSiO}_{4}$ 中的金属离子相连, 由此可 以提高膜基结合力 ${ }^{[16-17]}$, 获得致密硅酸锆薄膜。

\section{2 样品测试与表征}

采用联合热分析仪 (TG-DTA, NETZSCHSTA 449C)对热处理前的样品进行分析, 测试温度范围 为室温 $1200^{\circ} \mathrm{C}$, 升温速率为 $10^{\circ} \mathrm{C} / \mathrm{min}$ 。采用 $\mathrm{X}$ 射 线衍射仪(XRD, D8Advance)对样品进行物相分析。 通过场发射扫描电子显微镜(FESEM，JEM-6700F) 联合射线能量分析光谱仪(EDX, Hitachi SU-8010)对 样品的微观形貌进行分析表征; 使用原子力显微镜 (AFM, Micronano D3000 Atomic Force Microscope) 对比样品添加 APTES 前后显微形貌的变化。通过 NDJ-1 型旋转粘度计测定溶胶的粘度; 样品抗氧性 能测试是将试样置于空气气氛中以 $5^{\circ} \mathrm{C} / \mathrm{min}$ 升温速 率从室温升至 $1300^{\circ} \mathrm{C}$ 保温 $6 \mathrm{~h}$, 重复以上氧化过程 17 次并通过微量分析天平(Mettler Toledo, XPE26) 测试其质量变化过程 ${ }^{[18-19]}$ 。

\section{2 实验结果与讨论}

图 2 为 APTES 添加量分别为 $0 \mathrm{~mol} \% 、 5 \mathrm{~mol} \%$ 、 $10 \mathrm{~mol} \% 、 15 \mathrm{~mol} \%$ 和 $20 \mathrm{~mol} \%$ 时, 样品在 $1000^{\circ} \mathrm{C}$ 热

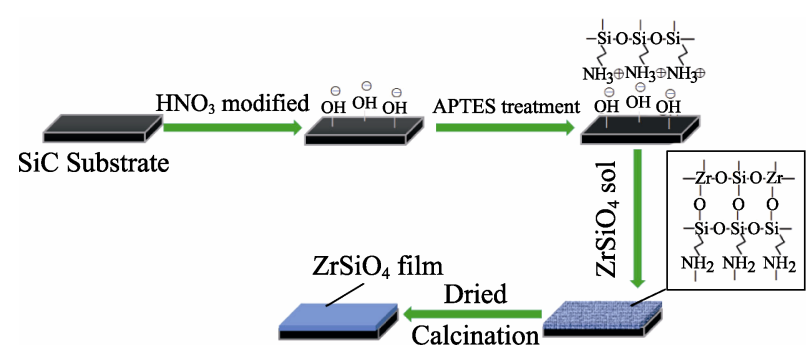

图 1 APTES 辅助溶胶一凝胶法制备 $\mathrm{ZrSiO}_{4}$ 薄膜的流程图 Fig.1 Schematic of the $\mathrm{ZrSiO}_{4}$ film prepared by APTES-assisted Sol-Gel method 
处理 $1 \mathrm{~h}$ 后的 XRD 图谱。随着 APTES 添加量逐渐 增大, 样品的衍射峰强度不断增强, 这是由于 APTES 在溶胶体系中发生了自水解缩聚反应, 产生 的 $\mathrm{SiO}_{2}$ 促进了 $\mathrm{ZrSiO}_{4}$ 的生成 ${ }^{[17]}$, 同时还可以补偿气 相 $\mathrm{SiF}_{4}$ 挥发后造成 $\mathrm{Si}$ 元素的缺失 ${ }^{[20]}$ 。当 APTES 添 加量增大至 $15 \mathrm{~mol} \%$ 和 $20 \mathrm{~mol} \%$ 时, 样品图谱中出现了 微弱的方石英衍射峰。为了避免 $\mathrm{SiO}_{2}$ 杂质相在升降温 过程发生相转变导致应力产生和薄膜开裂 ${ }^{[21]}$, APTES 的添加量应低于 $15 \mathrm{~mol} \%$ 。图 3 为添加 $10 \mathrm{~mol} \%$ APTES 前后样品的 DTA-TG 曲线, 从图中可以看出,

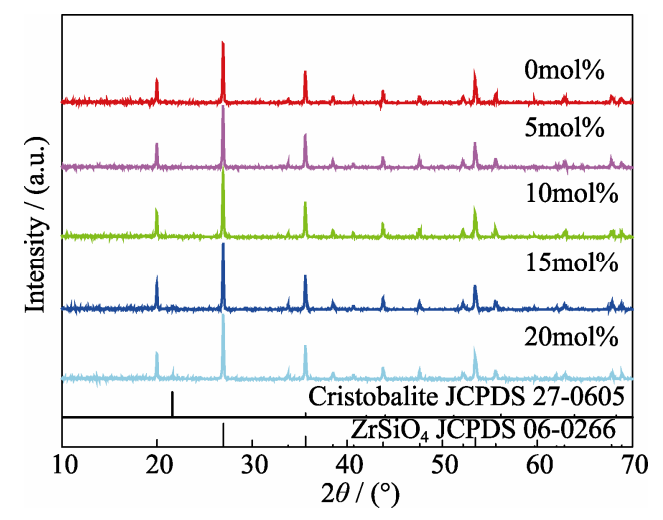

图 2 APTES 添加量对 $\mathrm{ZrSiO}_{4}$ 薄膜合成的影响

Fig. 2 Influence of APTES content on the synthesis of $\mathrm{ZrSiO}_{4}$ thin film
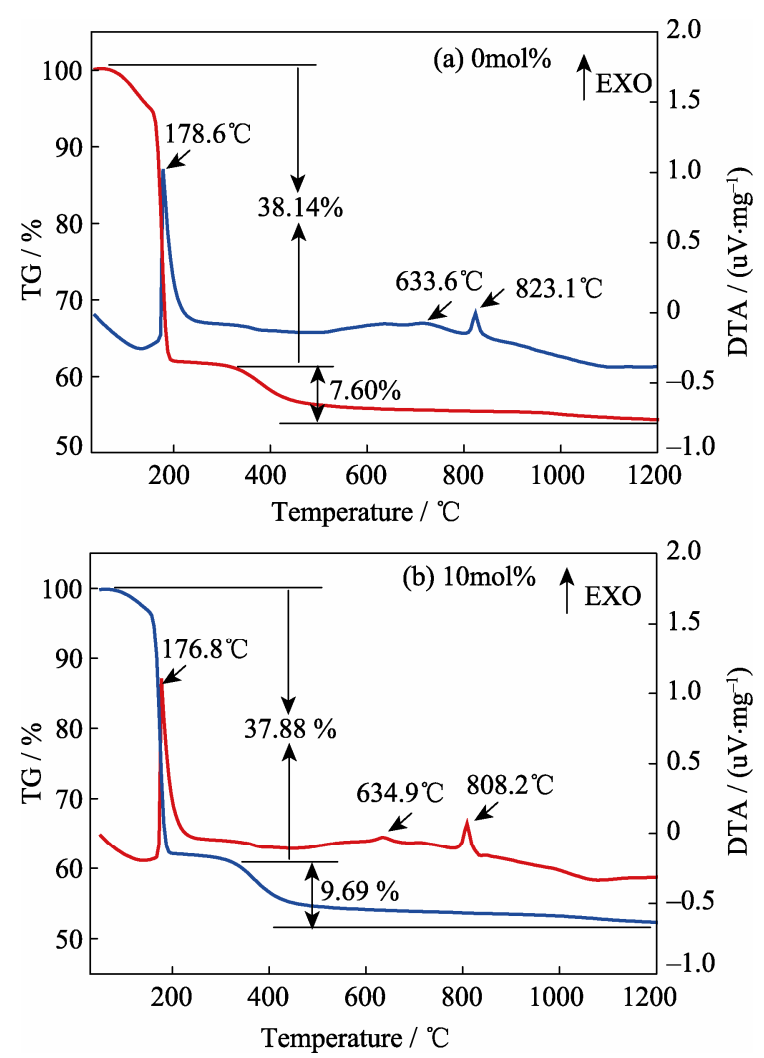

图 3 APTES 添加量对 $\mathrm{ZrSiO}_{4}$ 的 DTA-TG 分析结果的影响

Fig. 3 DTA-TG curves of $\mathrm{ZrSiO}_{4}$ samples with different APTES contents
添加 $10 \mathrm{~mol} \%$ 的 APTES 对样品的热失重无明显影响, 没有产生新相。APTES 添加量为 $0 \mathrm{~mol} \% 、 10 \mathrm{~mol} \%$ 、 $20 \mathrm{~mol} \%$ 时溶胶的黏度-时间曲线如图 4 所示, 加入 APTES 后, 溶胶的黏度明显上升, 这是由于 APTES 在 $\mathrm{HNO}_{3}$ 催化条件下自缩聚形成 $\mathrm{Si}-\mathrm{O}-\mathrm{Si}$ 网络 ${ }^{[22-24]}$ 。 尤其当 APTES 加入量为 $20 \mathrm{~mol} \%$ 时, 溶胶粘度上升 较快, 逐渐丧失流动性, 即成为凝胶, 而不利于均匀 成膜 ${ }^{[25]}$ 。综合以上实验结果说明 APTES 的最佳添加量 应为 $10 \mathrm{~mol} \%$ 。

图 5 为 $\mathrm{ZrSiO}_{4}$ 薄膜的 $\mathrm{AFM}$ 图像, 与未添加 APTES 的样品相比, 添加 $10 \mathrm{~mol} \%$ APTES 的薄膜表 面更为平滑致密，并且晶粒更细小。图 6 列出了添 加 APTES 制备的 $\mathrm{ZrSiO}_{4}$ 薄膜断面的面扫描分析和 线扫描分析结果, 其中, 蓝色、红色和绿色分别代表 了 $\mathrm{Zr} 、 \mathrm{O}$ 和 $\mathrm{Si}$ 元素。从图 6 可以看出, 添加 APTES 后可获得元素分布均匀的致密薄膜, 膜厚约为 $0.5 \mu \mathrm{m}$,

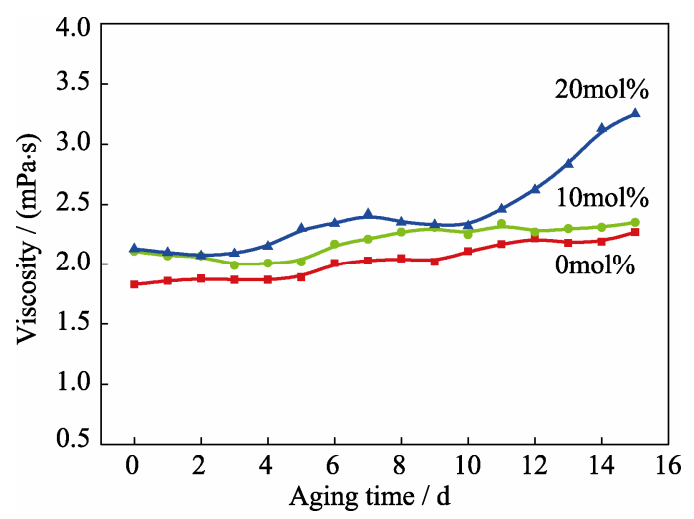

图 4 不同 APTES 添加量的溶胶黏度-时间曲线

Fig. 4 Time-viscosity curves of $\mathrm{ZrSiO}_{4}$ sol with various APTES contents

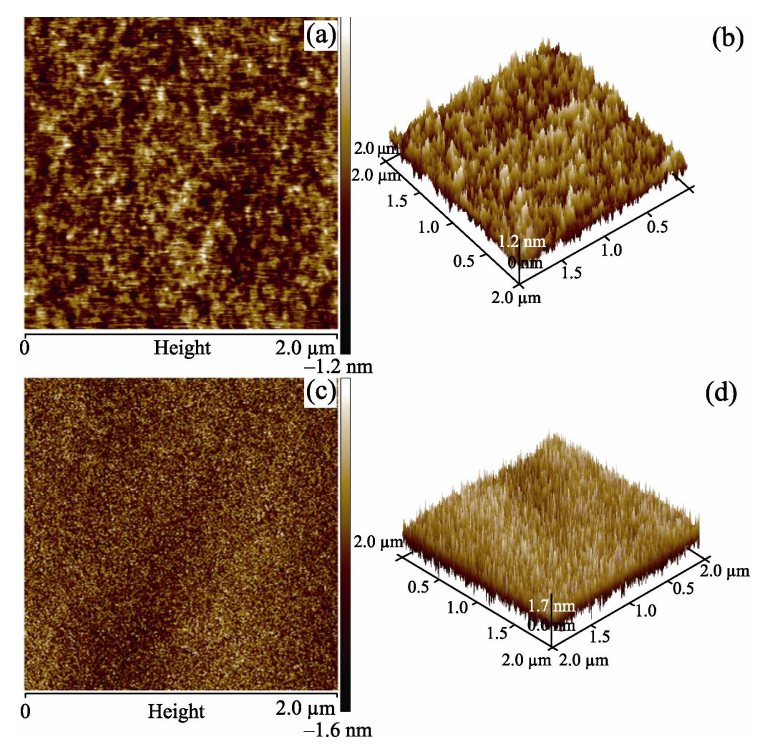

图 $5 \mathrm{ZrSiO}_{4}$ 薄膜的 $\mathrm{AFM}$ 图像

Fig. 5 AFM images of $\mathrm{ZrSiO}_{4}$ thin film (a, b) Without APTES; (c, d) With 10mol\% APTES 

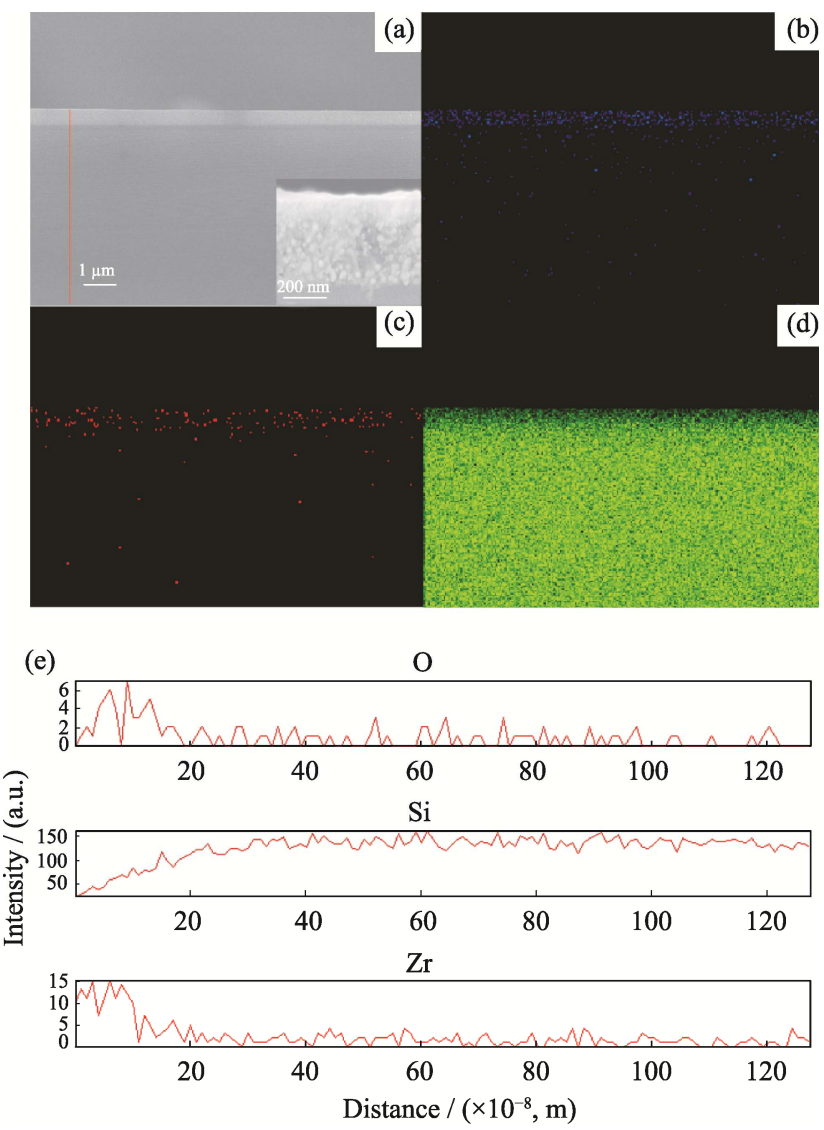

图 6 添加 APTES 制备的 $\mathrm{ZrSiO}_{4}$ 薄膜断面的面扫描分析结果

Fig. 6 Area scan analysis of the cross section of $\mathrm{ZrSiO}_{4}$ thin film with addition of APTES

(a) SEM; (b) Zr; (c) O; (d) Si; (e) Line scan analysis

硅酸锆晶粒粒径为 20 30 $\mathrm{nm}$ 。APTES 是一种硅醇 缩聚物，该类化合物在溶胶体系中易水解聚合，常 作为表面改性剂应用于 $\mathrm{NiS} @ \mathrm{SiO}_{2}{ }^{[26]} 、 \mathrm{C} @ \mathrm{ZrSiO}_{4}{ }^{[17]}$ 、 $\mathrm{CNT} @ \mathrm{SiO}_{2}{ }^{[24]}$ 等复合材料的制备。本研究中, APTES 结构中的氨基功能团与 $\mathrm{SiC}$ 基底表面带负电的羟基 产生静电吸附作用力 (图 1) ${ }^{[17,24,26]}$ 。同时, 乙酸锆与 正硅酸乙酯在酸催化条件下形成硅酸锆溶胶, 其中 的金属离子与 APTES 结构中的硅烷链形成 $\mathrm{Si}-\mathrm{O}-\mathrm{Si}$ 或 $\mathrm{Si}-\mathrm{O}-\mathrm{Zr}$ 键合 ${ }^{[17]}$, 有利于增大膜与基底的结合力, 提升胶体网络的强度, 进而提高薄膜的抗氧化能力。

煅烧后获得的致密硅酸锆薄膜可作为 $\mathrm{SiC}$ 基体 的抗氧化保护层，防止其高温下被氧化 ${ }^{[27-28]}$ 。图 7 给出了样品经 $1300^{\circ} \mathrm{C}$ 循环氧化 $102 \mathrm{~h}$ 后的质量变化 曲线, 其中单次氧化时间为 $6 \mathrm{~h}$ 。从图 7 可以看出, 未镀膜的 $\mathrm{SiC}$ 基底在氧化 $12 \mathrm{~h}$ 内迅速增重, 氧化 $102 \mathrm{~h}$ 质量增加了 $0.16 \%$, 此时 $\mathrm{SiC}$ 在高温下被氧化 成 $\mathrm{SiO}_{2}{ }^{[29]}$ 。相比之下, 添加 APTES 前后的样品的 质量变化分别为 $0.08 \%$ 和 $0.03 \%$, 表明 $\mathrm{ZrSiO}_{4}$ 薄膜 可以抵抗氧气对 $\mathrm{SiC}$ 基底的侵蚀。值得注意的是, 添 加 APTES 可以显著提高样品的抗氧化性能, 说明 添加 APTES 后制备的薄膜较为致密。抗氧化测试后, 将以上样品表面的 $\mathrm{ZrSiO}_{4}$ 薄膜小心打磨去除, 对 $\mathrm{SiC}$ 基体断面进行面扫描分析, 结果如图 8 所示。未
镀膜的 $\mathrm{SiC}$ 在高温下发生氧化反应，表面和内部均 生成了大量 $\mathrm{SiO}_{2}$ 。而镀膜后的 $\mathrm{SiC}$ 基底仅出现少量 的 $\mathrm{SiO}_{2}$, 添加 APTES 镀膜的 $\mathrm{SiC}$ 基底几乎未被氧化, 以上结果表明, 添加 APTES 可以提高样品的致密 度。样品在循环氧化 17 次后未出现明显的增重, 增 重量与采用等离子喷涂法制备的 $\mathrm{ZrSiO}_{4}$ 薄膜的 16 次循环氧化相近 ${ }^{[30]}$, 而且在 $\mathrm{SiC}$ 层中未观察到明显 的孔洞和裂纹 ${ }^{[31]}$, 说明采用溶胶一凝胶法制备的 $\mathrm{ZrSiO}_{4}$ 薄膜具有优异的抗氧化性能, 为制备高温热 障/热防护涂层材料提供了新的思路。

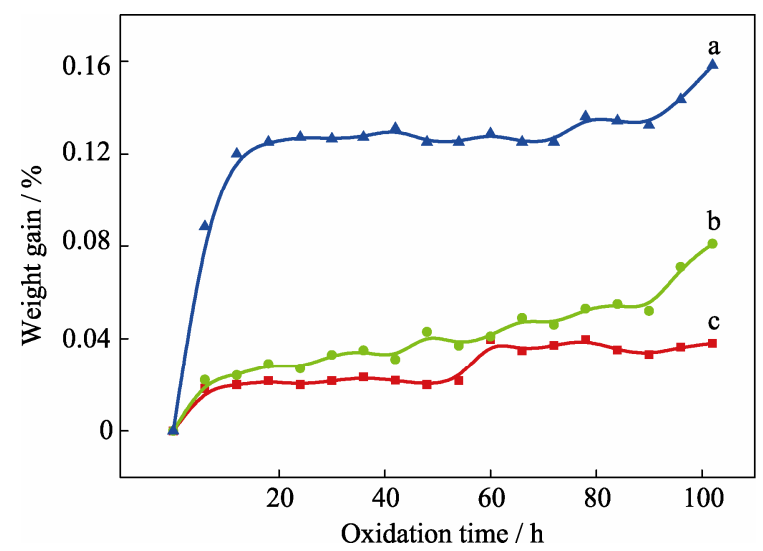

图 7 薄膜质量随氧化时间的变化曲线

Fig. 7 Change of thin film's weight with oxidation time (a) $\mathrm{SiC}$; (b) $\mathrm{SiC}$ coated by $\mathrm{ZrSiO}_{4}$ without APTES-assisted; (c) $\mathrm{SiC}$ coated by $\mathrm{ZrSiO}_{4}$ with APTES-assisted

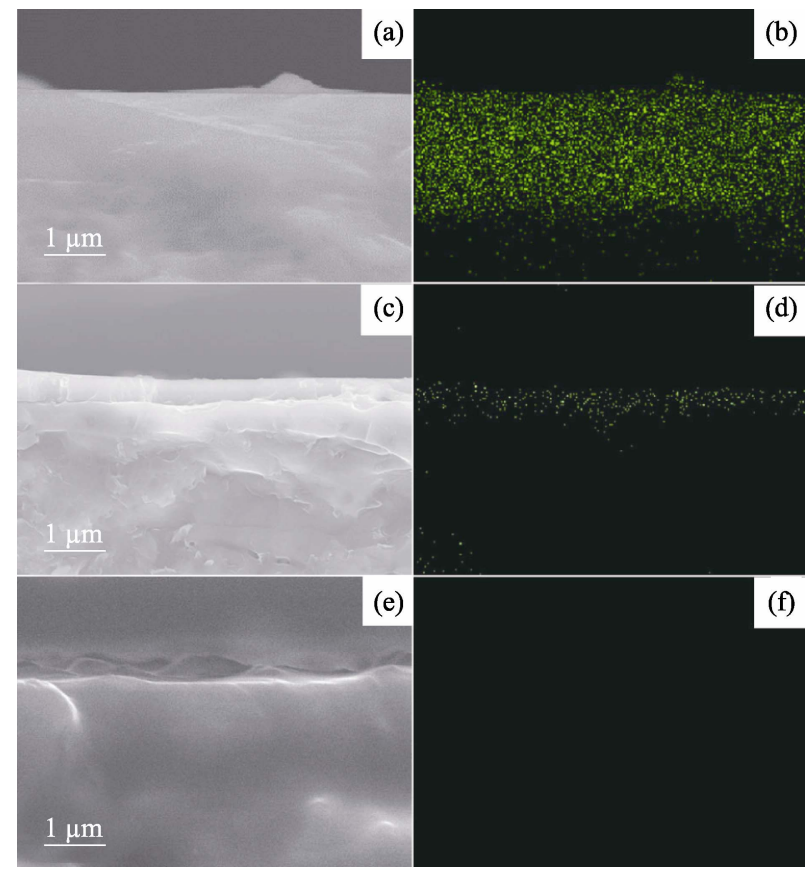

图 $81300^{\circ} \mathrm{C}$ 氧化后样品的 $\mathrm{SEM}$ 照片及其面扫描分析(O 元素) 结果

Fig. 8 SEM images and area scan analysis for $\mathrm{O}$ element of the cross section of the sample after oxidation at $1300^{\circ} \mathrm{C}$

(a) (b) $\mathrm{SiC}$; (c) (d) $\mathrm{SiC}$ coated by $\mathrm{ZrSiO}_{4}$ without addition of APTES; (e) (f) $\mathrm{SiC}$ coated by $\mathrm{ZrSiO}_{4}$ with addition of APTES 


\section{3 结论}

采用溶胶一凝胶法通过添加 $10 \mathrm{~mol} \%$ 的硅烷偶联 剂 APTES 可以制备出纯相的硅酸锆薄膜。对比未添 加 APTES 的薄膜, 添加 APTES 制备的硅酸锆薄膜 的表面更光滑致密。1300 ${ }^{\circ} \mathrm{C}$ 抗氧化测试 $102 \mathrm{~h}$ 后, 镀 膜的碳化硅基底的增重仅为 $0.03 \%$, 低于未镀膜的 $\mathrm{SiC}$ 基底 $(0.16 \%)$ 。抗氧化测试后样品的 $\mathrm{EDX}$ 分析结 果也证明, 添加 APTES 制备出的薄膜具有优异的 抗氧化性能。

采用溶胶一凝胶法制备的硅酸锆薄膜由于具有 良好的致密性和化学稳定性，有望替代硅成为金属一 氧化物一半导体器件中的闸极介电层。同时, 该膜还 具有与碳化硅基底良好的热匹配性、高熔点以及优 异的高温抗氧化性能, 因此在高温热障/热防护涂层 方面具有广阔的应用前景。

\section{参考文献:}

[1] GUO W M, XIAO H N, YAO X H, et al. Tuning pore structure of corrosion resistant solid-state-sintered $\mathrm{SiC}$ porous ceramics by particle size distribution and phase transformation. Mater. Des., 2016, 100: 1-7.

[2] PARK J Y. $\mathrm{SiC}_{\mathrm{f}} / \mathrm{SiC}$ Composites as Core Materials for Generation IV Nuclear Reactors. YVON P. Struct. Mater. Gener. IV Nucl. React., Netherlands: Elsevier, 2017: 441-470.

[3] FAN X Z, HUANG W Z, LIU H T, et al. Bond stability and oxidation resistance of BSAS-based coating on $\mathrm{C} / \mathrm{SiC}$ composites. Surf. Coat. Technol., 2017, 309: 35-46.

[4] LV Z L, LI S B, GAO J Q, et al. Effects of mullite coating on the anti-oxidation of silicon carbide at high temperature. Rare Metal Mat. Eng., 2003, 32(7): 534-537.

[5] LEMBERGER M, PASKALEVA A, ZÜRCHER S, et al. Zirconium silicate films obtained from novel MOCVD precursors. $J$. Non-Cryst. Solids, 2003, 322(1/2/3): 147-153.

[6] MARYAM H Z. Substitution of a fraction of zircon by cristobalite in nano hematite encapsulated pigment and examination of glaze application. J. Adv. Ceram., 2013, 2(2): 149-156.

[7] KAISER A, LOBERT M, TELLE R. Thermal stability of zircon $\left(\mathrm{ZrSiO}_{4}\right)$. J. Eur. Ceram. Soc., 2008, 28(11): 2199-2211.

[8] MORAIS J, ROSE E B O D, MIOTTI L, et al. Stability of zirconium silicate films on $\mathrm{Si}$ under vacuum and $\mathrm{O}_{2}$ annealing. Appl. Phys. Lett., 2001, 78(17): 2446-2448.

[9] LIU J, CAO L Y, HUANG J F, et al. A $\mathrm{ZrSiO}_{4} / \mathrm{SiC}_{\text {oxidation prot- }}$ ective coating for carbon/carbon composites. Surf. Coat. Technol., 2012, 206(14): 3270-3274.

[10] LIU J, CAO L Y, HUANG J F, et al. Influence of deposition temperature on microstructure of zirconium silicate coating prepared by hydrothermal electrophoretic deposition process. J. Aeronaut. Mater., 2011, 31(6): 30-35.

[11] ZHA J R, CHEN T, JIANG W H, et al. Preparation of $\mathrm{ZrSiO}_{4}$ thin film via Sol-Gel method. J. Ceram., 2016, 37(2): 133-139.

[12] YE L Q, ZHANG Y L, SONG C C, et al. A simple Sol-Gel method to prepare superhydrophilic silica coatings. Mater. Lett., 2017, 188: 316-318.

[13] SHI X H, XU K J. Properties of fluorine-doped tin oxide films prepared by an improved Sol-Gel process. Mater. Sci. Semicond.
Process., 2017, 58: 1-7.

[14] XIA S B, ZHANG Y J, DONG P, et al. High-performance $\mathrm{LiNi}_{0.8} \mathrm{Co}_{0.15} \mathrm{Al}_{0.05} \mathrm{O}_{2}$ cathode material prepared by Sol-Gel method in non-aqueous solvent. J. Inorg. Mater., 2015, 30(7): 732-738.

[15] LI M S, CHEN K W, WANG F H, et al. Structure and oxidation behavior of TiAlN coatings prepared by hollow cathode ion plating method. Corros. Sci. Prot. Technol., 2001, 13: 411-486.

[16] ZHANG Z H, HU Y F, ZHANG H B, et al. Layer-by-layer assembly sensitive electrochemical sensor for selectively probing L-histidine based on molecular imprinting Sol-Gel at functionalized indium tin oxide electrode. Biosens. Bioelectron., 2010, 26(2): 696-702.

[17] CHEN T, ZHANG X J, JIANG W H, et al. Synthesis and application of $\mathrm{C} @ \mathrm{ZrSiO}_{4}$ inclusion ceramic pigment from cotton cellulose as a colorant. J. Eur. Ceram. Soc., 2016, 36(7): 18111820 .

[18] MANOCHA L M, MANOCHA S M. Studies on solution-derived ceramic coatings for oxidation protection of carbon-carbon composites. Carbon, 1995, 33(4): 435-440.

[19] KANG N L, FOX D S, BANSAL N P. Rare earth silicate environmental barrier coatings for $\mathrm{SiC} / \mathrm{SiC}$ composites and $\mathrm{Si}_{3} \mathrm{~N}_{4}$ ceramics. J. Eur. Ceram. Soc., 2005, 25(10): 1705-1715.

[20] PENG C, ZHANG C X, LV M, et al. Co-precipitation synthesis and thermal stability of zircon encapsulated carbon black. J. Inorg. Mater., 2013, 28(8): 847-852.

[21] AKUTSU K, SAHARA M, MIYATA N. Study of a Corrosion Resistant Coating Resistant and Coating Layer for Metal Magnetic Cores Using Neutron Reflectometory. Proceedings of the 12th Annual Meeting of Particle Accelerator Society of Japan, Tsuruga, 2015: 71-74

[22] ABIDI N, CABRALES L, HAIGLER C H. Changes in the cell wall and cellulose content of developing cotton fibers investigated by FTIR spectroscopy. Carbohydr. Polym., 2014, 100: 9-16.

[23] GUNDA N S K, SINGH M, NORMAN L, et al. Optimization and characterization of biomolecule immobilization onsilicon substrates using (3-aminopropyl) triethoxysilane (APTES) and glutaraldehyde linker. Appl. Surf. Sci., 2014, 305: 522-530.

[24] ZHANG M, ZHANG X H, HE X W, et al. A facile method to coat mesoporous silica layer on carbon nanotubes by anionic surfactant. Mater. Lett., 2010, 64(12): 1383-1386.

[25] WANG J, ZHANG C R, FENG J, et al. Viscosity of $\mathrm{SiO}_{2}$ sol for preparing $\mathrm{SiO}_{2}$ aerogel film by Sol-Gel method. J. Nat. Univ. Defense Technol., 2003, 25(6): 30-34.

[26] ZHANG Z J, ZHAO H L, ZENG Z P, et al. Hierarchical architectured NiS@ $\mathrm{SiO}_{2}$ nanoparticles enveloped in graphene sheets as anode material for lithium ion batteries. Electrochim. Acta, 2015, 155: 85-92.

[27] YAMAMOTO O, SASAMOTO T, INAGAKI M. Antioxidation of carbon-carbon composites by $\mathrm{SiC}$ concentration gradient and zircon overcoating. Carbon, 1995, 33(4): 359-365.

[28] YAMAMOTO O, INAGAKI M. Antioxidation coating of carbon materials coupled with $\mathrm{SiC}$ concentration gradient. New Carbon Mater., 1999, 14(1): 1-7.

[29] POURASAD J, EHSANI N, KHALIFESOLTANI S A. Preparation and characterization of $\mathrm{SiO}_{2}$ thin film and $\mathrm{SiC}$ nanofibers to improve of graphite oxidation resistance. J. Eur. Ceram. Soc., 2016, 36(16): 3947-3956.

[30] SUN C, Li H J, LUO H J, et al. Effect of $\mathrm{Y}_{2} \mathrm{O}_{3}$ on the oxidation resistant of $\mathrm{ZrSiO}_{4} / \mathrm{SiC}$ coating prepared by supersonic plasma spraying technique for carbon/carbon composites. Surf. Coat. Technol., 2013, 235: 127-133.

[31] LI Y, ZHOU W, XIAO P, et al. The anti-oxidation behavior and infrared emissivity property of $\mathrm{SiC} / \mathrm{ZrSiO}_{4}-\mathrm{SiO}_{2}$ coating. J. Mater. Sci: Mater. Electron., 2014, 25(12): 5433-5440. 